\title{
Predicting Treatment Response from Resting State fMRI Data: Comparison of Parcellation Approaches
}

\author{
Satrajit S. Ghosh*, Anisha Keshavan* \\ * McGovern Institute for Brain Research \\ Massachusetts Institute of Technology \\ Cambridge, USA \\ satra@mit.edu,keshavan@mit.edu
}

\author{
Georg Langs $s^{\dagger \ddagger}$ \\ $\dagger$ CIR Lab, Department of Radiology \\ Medical University of Vienna, Vienna, Austria \\ ${ }^{\ddagger}$ Computer Science and Artifial Intelligence Lab \\ Massachusetts Institute of Technology, Cambridge, USA \\ georg.langs@meduniwien.ac.at
}

\begin{abstract}
Resting state fMRI reveals intrinsic network characteristics present in the brain. They are correlated with behavioral measures, and have made surprising insights in the brains' connectivity structure possible. At the core of many of those studies is the correlation of behavioral measures, and the characteristics of networks among a set of brain regions. In this paper we evaluate methods that identify functional networks in resting state fMRI in light of predicting treatment response of patients suffering from social anxiety disorder. Results illustrate differences in prediction when obtaining network labelings by population-wide-clustering, subject-specific parcellation, transferring anatomical region labels, or mapping networks from a previous large scale resting state study.
\end{abstract}

Keywords-Resting State Functional MRI; Diffusion Embedding; Graph Measures; Treatment outcome

\section{INTRODUCTION}

The human cerebral system is characterized by a complex network architecure. It can be observed in functional magnetic resonance imaging data acquired during rest (rsfMRI). Networks exhibit remarkable consistency across the population [1], and some of their properties were reported to correlate with cognitive capabilities [2]. The precise link between cognition and network structure is currently only poorly understood. This has triggered active research aiming at a reliable identification of functional networks from rsfMRI data, their quantitative characterization and its use as potential predictor for behavioral measurements, disease, or even treatment outcome.

A common approach to network segmentation in rs-fMRI is independent component analysis (ICA) [3], [4]. Clustering approaches [1] have recently been adopted, and can identify networks in large populations based on similarity measures that typically view the image voxels, or cortical surface points as a graph. Alternatives are sparse learning approaches [5] that parcellate the brain relying on sparsity constraints. These techniques assume spatial consistency across the study population placing functional units at the exact same position in each individual of the population. In [6] this constraint was relaxed, and a method for the group-wise registration of multiple subjects based on their fMRI signals, instead of anatomy was described. After parcellation different approaches have been put forward to quantify the characteristics of the network or graph whose nodes are formed by the parcels. [7] provides an extensive review of a number of relevant approaches that capture properties such as small world-ness of networks, or features of individual nodes.

Currently the differences among the individual parcellation approaches, and their impact on neuroscientific findings is not entirely understood. Furthermore, in the absence of ground truth, the performance, importance, or relevance of each parcellation is often difficult to determine. In this paper we compare 5 methods for brain parcellation of rs-fMRI data. We compare their performance by predicting individual treatment response in social anxiety disorder (SAD). We use the graph formed by the resulting parcels as a basis for the predictor.

We describe the experiment data in Sec. II, and outline the individual approaches briefly in Sec. III. In Sec. IV we explain the prediction procedure, and in Sec. V we report quantitative results.

\section{Collecting MeAsurements}

We evaluated the clustering approaches, and the effect of functional alignment on rs-fMRI data acquired during a clinical treatment study of social anxiety disorder ( $\mathrm{n}=35$; approved by MIT Institutional Review Board [8]). The BOLD EPI data (TR=6s, $2 \mathrm{~mm}$ isometric voxels, 6 mins, interleaved acquisition, whole head coverage) were preprocessed using the BIPS framework built using Nipype [9] and involved simultaneous slicetiming and motion correction [10], nuisance regression using estimated motion parameters, artifactual timepoints, anatomical component correlation (CompCor; [11]) and surface-based smoothing of the data. Since no dummy scans were acquired, the first 5 timepoints of the functional run were discarded to maximize magnetic equilibrium. The mean functional image was registered using bbregister [12] to the participant's FreeSurfer surface. This registration was used in combination with the default spherical registration to sample the preprocessed timeseries onto an average FreeSurfer surface (fsaverage4). 


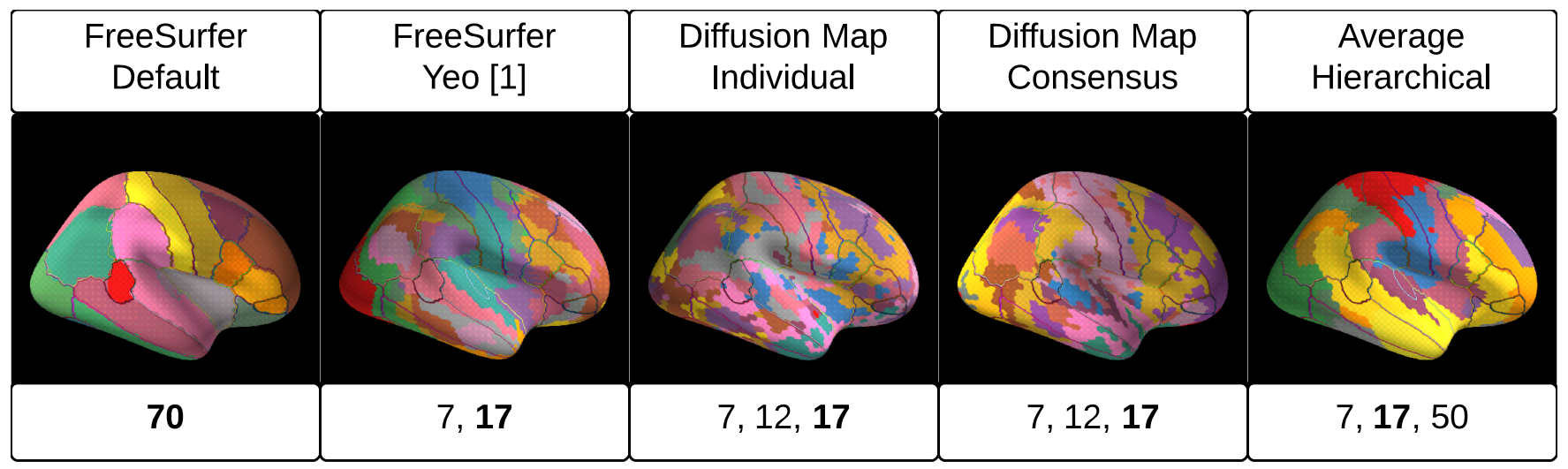

Figure 1. Cortical surface labels. The top row shows examples of the 5 parcellation schemes used to determine the correlation matrices. The bottom row shows the different numbers of clusters that were selected for each parcellation type. The number in bold indicates the number of clusters in the displayed parcellation. The outlines of the automatically parcellated FreeSurfer regions (leftmost scheme) are shown on each figure.

\section{Clustering Resting STATE FMRI DATA}

All 5 parcellation generation algorithms are given one fMRI sequence $\mathbf{I}_{i} \in \mathbb{R}^{T \times N}$ for each subject $i$. $\mathbf{I}_{i}$ contains $N$ cortical surface vertices each characterized by an fMRI signal over $T$ time points. Parcellation aims at either assigning population wide parcel labels $l_{n}$, or subject specific labels $l_{n}^{i}$. In the following we briefly outline the parcellation approaches (Fig. 1) compared in this paper.

\section{A. FreeSurfer automatic parcellation}

The FreeSurfer (version 5.1) Desikan-Killiany parcellation was used to define labels $l_{n}$ on the surface for each individual [13]. This is performed as part of the standard FreeSurfer 'recon-all' processing stream.

\section{B. Cortico Cortical Profiles}

We used two resting state networks comprising 7 and 17 nodes defined in [1] to label every individual's cortical surface. They are based on clustering surface voxels based on the correlation of their signal with those at a fixed set of anchorpoints distributed across the cortex. This labeling $l_{n}$ was applied to our data using 'mri_surf2surf' and the FreeSurfer spherical registration that is automatically computed between an individual's surface and the FreeSurfer average subject as part of the standard FreeSurfer preprocessing.

\section{Clustering after alignment of subject embeddings}

For subject-specific parcellation, we performed functional alignment as introduced in [6] before clustering in the joint embedding space. In this approach the fMRI data is represented by an affinity matrix based on the correlation coefficient of the time course pairs $\mathbf{I}_{i}$ and $\mathbf{I}_{j}$. It defines a graph whose vertices correspond to cortical surface points and whose edge weights are determined by $\mathbf{W}$ [14].
The corresponding Markov transition matrix determines a diffusion distance among all points. A low dimensional embedding maps all voxels of each subject $i$ to coordinates $\boldsymbol{\Gamma}_{i}=\left[\gamma_{1}, \gamma_{2}, \cdots, \gamma_{N}\right]^{T}$ yields a map in which the Euclidean distance approximates the diffusion distance in the graph. The embedding coordinates of multiple subjects are then orthonormally aligned to obtain a representation of all voxels of all subjects in the embedding space $\Gamma$.

To identify clusters in the population data, the algorithm clusters $\boldsymbol{\Gamma}$ by a Gaussian mixture model $k$ components, and diagonal covariance matrices. After clustering the cluster labels are mapped back to the individual subjects, by assigning all voxels corresponding to the embedding points in cluster the corresponding label. This results in subject specific cluster labels $l_{n}^{i}$, that are learned based on the entire population.

\section{Consensus of embedding space clusters}

We can transform the subject-specific clustering described in the previous section into a unified labeling of the population by forming the consensus labels of each cortical surface point. It unifies cluster assignments on the cortical surface across the population. The cluster label $l_{n}$ of each surface vertex is determined by majority vote of the cluster labels assigned to this vertex throughout the population.

\section{E. Hierarchical clustering of diffusion embedding}

Another way of generating a consensus clustering is to derive it from the average correlation matrix. We repeat the same diffusion embedding procedure to define a smaller subspace (vertices $\times$ components). On this subspace we perform hierarchical clustering using the Ward algorithm. In order to compare with the previous approaches, we generate 7, 17 and 50 parcels, by cutting the dendogram generated by the clustering algorithm at appropriate levels. 
For each labeling or parcellation of an individual's cortical surface, we computed the mean time course of each label and then computed the correlation between the mean timecourses of every pair of labels to create a symmetric matrix.

\section{PREDicting TREATMENT RESPONSE}

Pre-treatment correlations, graph measures and behavioral scores (Liebowitz Social Anxiety Scale - LSAS-Pre) were used to predict post-treatment LSAS score (LSAS-Post), change (LSAS- $\Delta$ ) or percentage change (LSAS- $\Delta$ /LSASPre). All three of these outcome options would be useful to the clinician although the functions mapping correlations or network measures to these variables may be quite different. The correlation matrices were used in raw form (as edge weights between label nodes) or to compute weighted measures (e.g., average neighbor degree) and binary measures (betweenness centrality and degree centrality) using the Networkx library [15] although many of these algorithms are available in the Brain Connectivity Toolbox [16] as well. The binary measures were computed by thresholding the correlation matrix.

\section{A. Using correlations to find significant edges}

For each network, we calculated the correlation between any single edge in the brain label network and the treatment outcome. No edges survived corrections for multiple comparisons. Therefore, we used this as an exploratory feature to relate regions that showed significant $(p<0.05$; uncorrected) connections. An example of such an approach is shown in Figure 2.

\section{B. Using graph measures to predict outcome}

For each network, we used scikit.learn [17] to determine the median explained variance score when the network features (i.e. graph measures) are used to predict outcome. This was performed using a random sampling of test and train samples over 1000 iterations. For the purpose of this paper, we restricted the model to Ridge Regression. The regularization parameter for the regression was chosen using an inner 3-fold cross-validation loop.

\section{Results}

Table I summarizes the results of predicting LSAS $\Delta$, LSAS $\Delta \%$, and LSAS post based on the pre treatment rs-fMRI data. We compare 4 parcellations: Clusters on a different population [1] (CP), hiearchical clustering of diffusion embeddings (HIE), Freesurfer cortex parcellation [13] (FS), and clustering in the joint embedding space [6] (JEMB). We report the variance explained by the prediction in the cross-validation experiment. Although not consistent across all experiments in general lower numbers of parcels tend to coincide with better prediction accuracy. Overall, prediction results are best for LSAS post, followed by LSAS $\Delta$. HIE and $\mathrm{CP}$ yield the best overall results 0.20 for

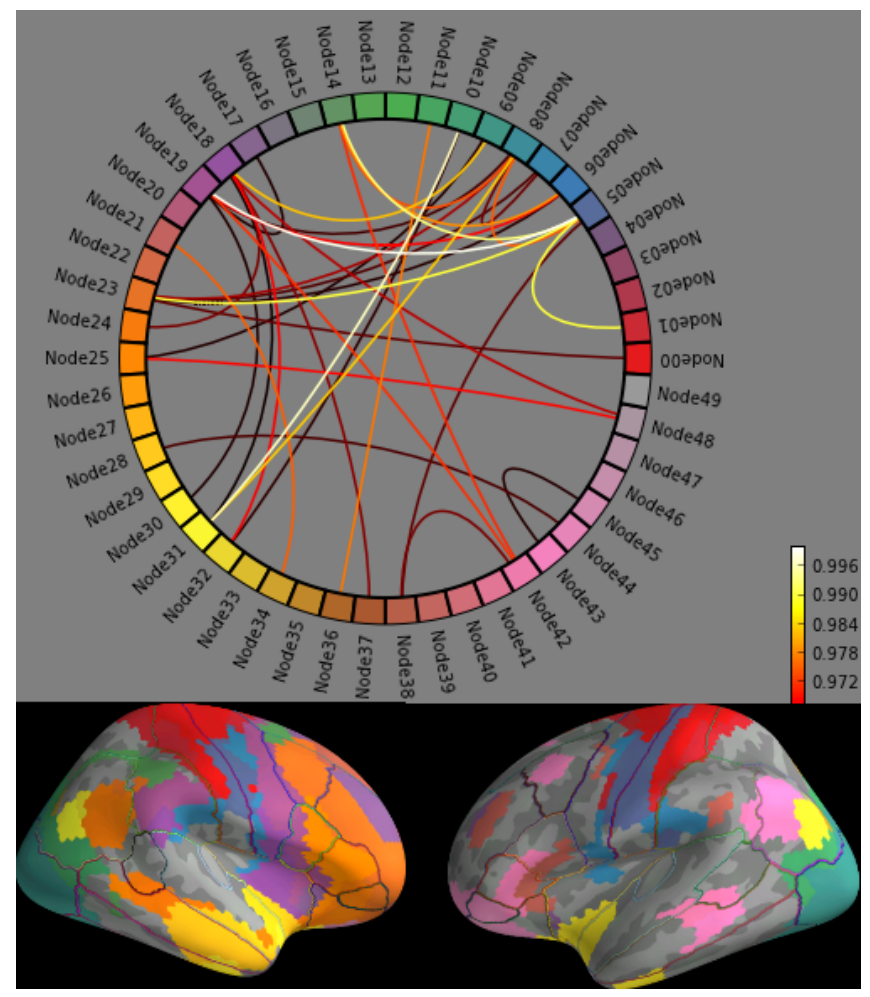

Figure 2. Correlation example. Significant regions found for an example correlation of matrix elements or edges with treatment outcome. Line color reflects $1-p$.

\begin{tabular}{|l|l|rrr|}
\hline Parcellation & K & LSAS $\Delta$ & LSAS $\Delta \%$ & LSAS post \\
\hline CP [1] & 7 & 0.16 & 0.06 & $\mathbf{0 . 2 0}$ \\
CP [1] & 17 & 0.06 & -0.03 & 0.11 \\
\hline HIE average & 7 & 0.12 & 0.04 & $\mathbf{0 . 2 0}$ \\
HIE average & 17 & 0.16 & -0.01 & 0.12 \\
HIE average & 50 & -0.70 & -0.81 & -0.65 \\
\hline FS & 70 & -1.57 & -1.51 & -1.3 \\
\hline JEMB consensus & 7 & 0.17 & 0.04 & 0.19 \\
JEMB individual & 7 & 0.17 & 0.10 & 0.19 \\
JEMB consensus & 12 & 0.02 & -0.05 & 0.04 \\
JEMB individual & 12 & 0.12 & 0.03 & 0.12 \\
JEMB consensus & 17 & 0.02 & 0.01 & 0.13 \\
JEMB individual & 17 & 0.19 & 0.03 & 0.17 \\
\hline
\end{tabular}

Table I

CROSS-VALIDATED EXPLAINED VARIANCE FOR LSAS POST, LSAS CHANGE, AND LSAS PERCENTAGE CHANGE ACHIEVED WITH DIFFERENT PARCELLATIONS. PARCELLATIONS COMPRISE PARCELS FROM [1] (CP), HIEARCHICAL CLUSTERING OF DIFFUSION EMBEDDING (HIE), FREESURFER CORTEX PARCELLATION [13] (FS), AND CLUSTERING IN THE JOINT EMBEDDING SPACE [6] (JEMB).

LSAS post prediction. For both $\Delta$ predictions JEMB with 7 clusters and aligned but subject specific parcels perform best. Across all tasks, and numbers of parcels, aligned subject specific parcels outperform static consensus parcels for JEMB. Freesurfer parcels perform poorly across all three prediction tasks. 


\section{CONCLUSION}

Our results demonstrate the differences in prediction when using different approaches for the label generation and assignment, each of which are equally likely to be in use in brain imaging. We use correlation between intrinsic connectivity of the brain and treatment outcome as a measure of successful registration, based on the assumption that improved predictive power of the measurements across subjects stems from a more accurate registration of "functionally conncected" areas across subjects. We assume that a decrease of predictive power, stems from misalignment, and thus a loss of information. In the absence of ground truth, comparing prediction power provides one possible approach for evaluating the efficacy of the methods. However, these labels are derived from specific settings on distance metrics, diffusion time and choices of subspace size. To evaluate these parameter choices exhaustively and without incurring too much bias in such clinical contexts will require significantly larger data sets. Results highlight that the granularity of the 'true' networks might be substantially higher than we are able to capture by individual clustering approaches. The matching between clusters and networks might vary across the cortex.

Future work will be aimed at assessing the stability of these label derived prediction frameworks in the context of large clinical intervention data.

\section{ACKNOWLEDGMENT}

Data were collected from a subset of participants who took part in a clinical trial registered at clinicaltrials.gov (NCT00515879), with MIT IRB authorization and the brain imaging was performed in Athinoula A. Martinos Imaging Center at McGovern Institute for Brain Research, MIT.

\section{REFERENCES}

[1] B. T. T. Yeo, F. M. Krienen, J. Sepulcre, M. R. Sabuncu, D. Lashkari, M. Hollinshead, J. L. Roffman, J. W. Smoller, L. Zöllei, J. R. Polimeni, B. Fischl, H. Liu, and R. L. Buckner, "The organization of the human cerebral cortex estimated by intrinsic functional connectivity," J Neurophysiol, vol. 106, no. 3, pp. 1125-65, Sep 2011.

[2] M. P. van den Heuvel, C. J. Stam, R. S. Kahn, and H. E. Hulshoff Pol, "Efficiency of functional brain networks and intellectual performance," J Neurosci, vol. 29, no. 23, pp. 7619-24, Jun 2009.

[3] V. D. Calhoun, J. Liu, and T. AdalI, "A review of group ica for fmri data and ica for joint inference of imaging, genetic, and erp data," NeuroImage, vol. 45, no. 1, Supplement 1, pp. S163 - S172, 2009, mathematics in Brain Imaging. [Online]. Available: http://www.sciencedirect.com/science/article/B6WNP4TX7943-7/2/c0deb2b194ee0f47da1b9d42cd991aaa

[4] V. Schöpf, C. Windischberger, S. Robinson, C. H. Kasess, F. P. Fischmeister, R. Lanzenberger, J. Albrecht, A. M. Kleemann, R. Kopietz, M. Wiesmann, and E. Moser, "Modelfree fmri group analysis using fenica," Neuroimage, vol. 55, no. 1, pp. 185-93, Mar 2011.
[5] G. Varoquaux, A. Gramfort, F. Pedregosa, V. Michel, and B. Thirion, "Multi-subject dictionary learning to segment an atlas of brain spontaneous activity," Inf Process Med Imaging, vol. 22, pp. 562-73, 2011.

[6] G. Langs, D. Lashkari, A. Sweet, Y. Tie, L. Rigolo, A. J. Golby, and P. Golland, "Learning an atlas of a cognitive process in its functional geometry," Inf Process Med Imaging, vol. 22 , pp. 135-46, 2011.

[7] E. Bullmore and O. Sporns, "Complex brain networks: graph theoretical analysis of structural and functional systems," Nat Rev Neurosci, vol. 10, no. 3, pp. 186-98, Mar 2009.

[8] O. Doehrmann, S. S. Ghosh, F. E. Polli, G. O. Reynolds, F. Horn, A. Keshavan, C. Triantafyllou, Z. M. Saygin, S. Whitfield-Gabrieli, S. G. Hofmann, M. Pollack, and J. D. Gabrieli, "Predicting treatment response in social anxiety disorder from functional magnetic resonance imaging." Arch Gen Psychiatry, pp. 1-11, 92012.

[9] K. Gorgolewski, C. D. Burns, C. Madison, D. Clark, Y. O. Halchenko, M. L. Waskom, and S. S. Ghosh, "Nipype: a flexible, lightweight and extensible neuroimaging data processing framework in python," Frontiers in neuroinformatics, vol. 5, 2011.

[10] A. Roche, "A four-dimensional registration algorithm with application to joint correction of motion and slice timing in fmri," Medical Imaging, IEEE Transactions on, vol. 30, no. 8, pp. 1546-1554, 2011.

[11] Y. Behzadi, K. Restom, J. Liau, and T. Liu, "A component based noise correction method (compcor) for bold and perfusion based fmri," Neuroimage, vol. 37, no. 1, p. 90, 2007.

[12] D. N. Greve and B. Fischl, "Accurate and robust brain image alignment using boundary-based registration," Neuroimage, vol. 48, no. 1, p. 63, 2009.

[13] R. S. Desikan, F. Ségonne, B. Fischl, B. T. Quinn, B. C. Dickerson, D. Blacker, R. L. Buckner, A. M. Dale, R. P. Maguire, B. T. Hyman, M. S. Albert, and R. J. Killiany, "An automated labeling system for subdividing the human cerebral cortex on mri scans into gyral based regions of interest," Neuroimage, vol. 31, no. 3, pp. 968-80, Jul 2006.

[14] R. R. Coifman and S. Lafon, "Diffusion maps," App. Comp. Harm. An., vol. 21, pp. 5-30, 2006.

[15] A. A. Hagberg, D. A. Schult, and P. J. Swart, "Exploring network structure, dynamics, and function using NetworkX," in Proceedings of the 7th Python in Science Conference (SciPy2008), Pasadena, CA USA, Aug. 2008, pp. 11-15.

[16] M. Rubinov and O. Sporns, "Complex network measures of brain connectivity: uses and interpretations," Neuroimage, vol. 52, no. 3, pp. 1059-1069, 2010.

[17] F. Pedregosa, G. Varoquaux, A. Gramfort, V. Michel, B. Thirion, O. Grisel, M. Blondel, P. Prettenhofer, R. Weiss, V. Dubourg et al., "Scikit-learn: Machine learning in python," The Journal of Machine Learning Research, vol. 12, pp. 2825-2830, 2011. 